\title{
HIGH TIBIAL CLOSED WEDGE OSTEOTOMY FOR MEDIAL COMPARTMENTAL KNEE ARTHROSIS
}

\author{
Satya Kumar Koduruํ, Srinivas Raghav Thota², Siddhartha Potluri ${ }^{3}$
}

1 Professor, Department of Orthopaedics, NRI Academy of Sciences, Guntur, Andhra Pradesh. ${ }^{2}$ Senior Resident, Department of Orthopaedics, NRI Academy of Sciences, Guntur, Andhra Pradesh. 3Junior Resident, Department of Orthopaedics, NRI Academy of Sciences, Guntur, Andhra Pradesh.

\begin{tabular}{l}
\hline ABSTRACT \\
AIMS \\
To study the outcome of high tibial osteotomy in medial compartment arthritis. \\
SETTINGS AND DESIGN \\
$\quad$ Prospective as well as retrospective study of thirty six patients with unicompartmental osteoarthritis of the knee presenting to \\
our hospital from the year 2013 to 2015 , who were treated with high tibial osteotomy.
\end{tabular}

\section{METHODS AND MATERIAL}

The study material includes data collection, clinical examination and investigations of thirty six patients who underwent high tibial osteotomy. Out of thirty six patients, twenty four patients were women and twelve patients were men with a ratio of 2:1.

The mean age at the time of surgery was 54.44 years with a range of 33 years to 73 years.

Right side unicompartmental osteoarthrosis is seen in seventeen patients (41.67\%) and nineteen patients (58.33\%) had left side involvement.

\section{RESULTS}

A $75 \%$ patients had $90 \%$ to $100 \%$ of pain relief. Two fold increase in the number of patients with pain free unlimited walking distance. All patients had an improvement in their knee score. Over $90 \%$ of patients were free of complications.

\section{CONCLUSIONS}

High tibial osteotomy remains a useful and efficient procedure. The improvement in the post op scoring encourages us to continue recommending HTO in the most painful early and medium stages of medial compartment osteoarthritis.

HTO is thus very beneficial in cases of a good patient selection and good operative technique has been achieved.

\section{KEYWORDS}

Osteotomy, Varus, Osteoarthritis.

HOW TO CITE THIS ARTICLE: Koduru SK, Thota SR, Potluri S. High tibial closed wedge osteotomy for medial compartmental knee arthrosis. J Evolution Med Dent Sci 2016;5(1):66-70, DOI: 10.14260/jemds/2016/16

\section{INTRODUCTION}

High tibial osteotomy distributes the stress of weight bearing across the knee more evenly and relieves excessive pressure. Most patients of symptomatic osteoarthrosis of knee are associated with varus malalignment that is causative or contributory factor to painful arthrosis. The goals of osteotomy are to relieve pain, to redistribute weight bearing forces, to improve function and thereby potentially increase the longevity of the native knee joint. ${ }^{1}$ this study, reports the outcome of high tibial osteotomy in the medial compartment osteoarthrosis of the knee done in our hospital.

The overall HTO results show the effectiveness of the procedure. ${ }^{2}$ in the active patient with a life expectancy of twenty years or more with unicompartmental osteoarthrosis, high tibial osteotomy is a generally accepted treatment that can result in excellent pain relief and functional improvement.

Financial or Other, Competing Interest: None.

Submission 15-12-2015, Peer Review 16-12-2015,

Acceptance 30-12-2015, Published 04-01-2016.

Corresponding Author:

Dr. K. Satya Kumar

Flat No. 1,

Kanchukota N. K. Habitate, Gayatri Nagar,

Vijayawada-520008,

Andhra Pradesh.

E-mail: kodurusk@gmail.com

DOI:10.14260/jemds/2016/16

\section{MATERIALS AND METHODS}

In this retrospective as well as prospective study of thirty six patients with unicompartmental osteoarthrosis of the knee presenting to our hospital from year 2013 to 2015 were treated with high tibial osteotomy.

\section{MATERIALS}

The study material includes data collection, clinical examination and investigations of thirty six patients who underwent high tibial osteotomy. Out of thirty six patients, twenty four patients were women and twelve patients were men with a ratio of $2: 1$. The mean age at the time of surgery was 54.44years with a range of 33 years to 73 years.

Right side unicompartmental osteoarthrosis is seen in seventeen patients (41.67\%) and nineteen patients (58.33\%) had left side involvement.

\section{Inclusion Criteria}

Patients with medial compartmental osteoarthrosis.

Patients with Prides criteria (Radiological) type I and II.

Patients with minimum 1 year 3 months follow up.

\section{Exclusion Criteria}

Patients with pre-existing disease or trauma to the knee.

Patients with polyarticular involvement. 
Grossly unstable knee.

Preoperative Planning

Patient's problem was evaluated using,

1. Clinical functional criteria.

2. Radiological criteria.

\section{CLINICAL FUNCTIONAL CRITERIA}

The parameters which were assessed in clinical and functional criteria are Pain, Range of motion, Walking distance, Coventry's rating system, Insall's modified knee score.

\section{Radiological Criteria}

Tibiofemoral angle correction.

\section{Operative Procedure}

Under spinal anesthesia, patient in supine position, the limb was prepared and draped from foot to thigh and a tourniquet was used. The procedure is carried out under image intensifier control. The affected limb was placed at an angle of $90^{\circ}$ this carries the popliteal vessels and the peroneal nerve posteriorly and relaxes the iliotibial band 3 bony points, viz. the head of fibula and the tibial tubercle and Gerdy's tubercle are marked. An oblique incision between these three bony landmarks starting from tibial shin to mid lateral aspect of the knee joint and ended just proximal to the lateral femoral condyle.

The anterior tibial muscles are dissected stripped from the tibia up to the proximal $1 / 3$ of tibial taking care not to injure the anterior tibial vessels which pass along the anterior compartment. Tibiofibular syndesmosis identified and release done. Parallel to joint line and $1.5 \mathrm{~cm}$ distally is drilled and cancellous screw with washer is passed parallel to the joint line and a cortical screw passed distally in the same line. The osteotomy was carried out under direct vision using a wide osteotome using power saw. The upper limit of the wedge was first marked out along a plane two centimeters below and parallel to the articular surface. The appropriate wedge was marked out below the previously established line. Wedge is removed leaving a thin posteromedial lip of bone on the proximal tibial fragment following closure of the osteotomy after removal of wedge of bone.

The posterior lip overrides the proximal end of the distal fragment and thus gives added support and stability to osteotomy. By this method the distal fragment is displaced anteriorly there by obtaining the Maquet effect.

The osteotomy is approximated with lateral cerclage in figure of eight. Keeping the in valgus the corrected knee is checked under image intensifier.

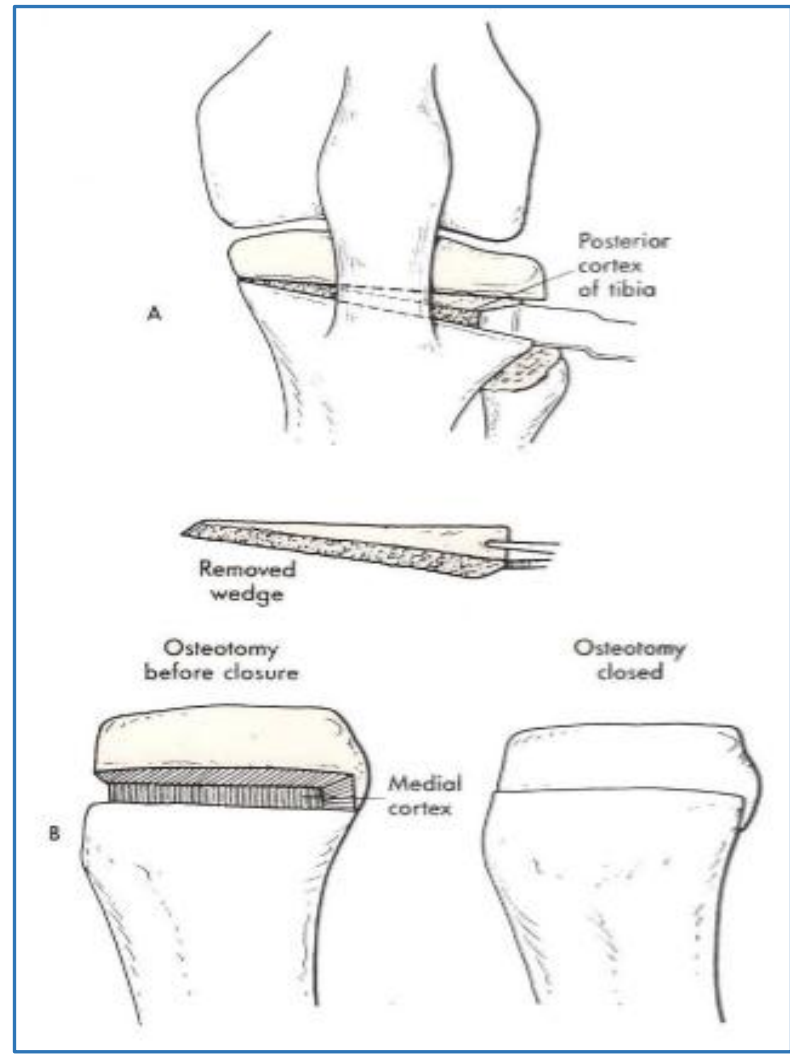

A, Bone wedge is cut, but not through posterior cortex of tibia before its removal. B, Lower saw cut is deepened through posterior cortex and osteotomy wedge closed as illustrated on right. This permits posterior lip of bone above to override cortex distal to osteotomy for stability.

\section{Post-operative Protocol}

Postoperatively intravenous antibiotics given for three days followed by oral antibiotics for another week. Wound inspection is done on second post-operative day and drain is removed. Suture removal is done on eleventh post-operative day. An antero-posterior, lateral radiographs and an orthoscanograms were taken alignment was assessed.

\section{Physiotherapy}

Active and passive knee mobilization was done on first postoperative day. Quadriceps and hamstring exercises were taught and allowed to practice under supervision.

\section{Mobilization}

Full weight bear mobilization was started on second postoperative day after teaching active quadriceps exercises.

\section{Post-operative Tibiofemoral Angulation}

Postoperative tibio-femoral angle is calculated using radiographs and orthoscanograms.

Patients followed up for every six weeks and clinical examinations, radiographs were taken in antero posterior, lateral in standing position and the joint space, any regression in the varus angle and the tibio femoral alignment is looked after.

\section{RESULTS}

\section{Percentage of Pain Relief}

Percentage of pain relief is measured using visual analog scale and percentage of pain calculated accordingly. 


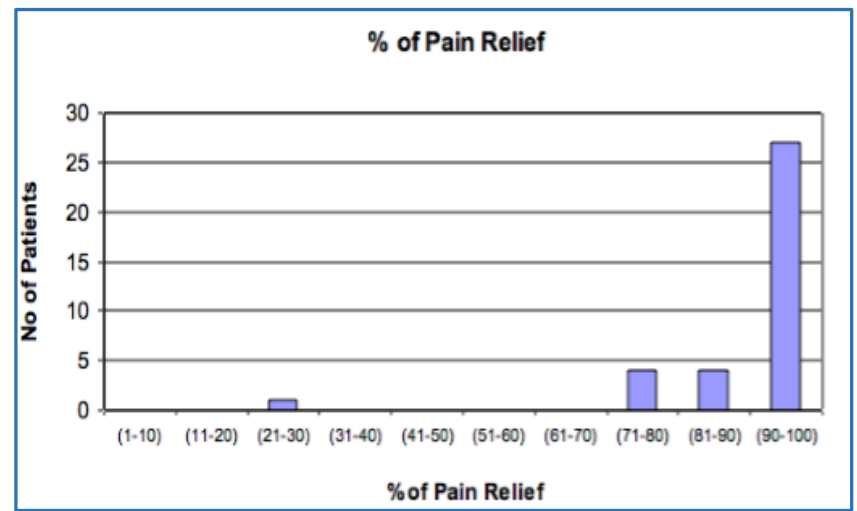

Fig. 1: Percentage of Pain Relief

\section{Range of Motion}

The arc of motion was in range of 0 to $120^{\circ}$. All most all knees had greater than $90^{\circ}$ of flexion. The range of motion assessed with goniometer showed significant improvement post operatively.

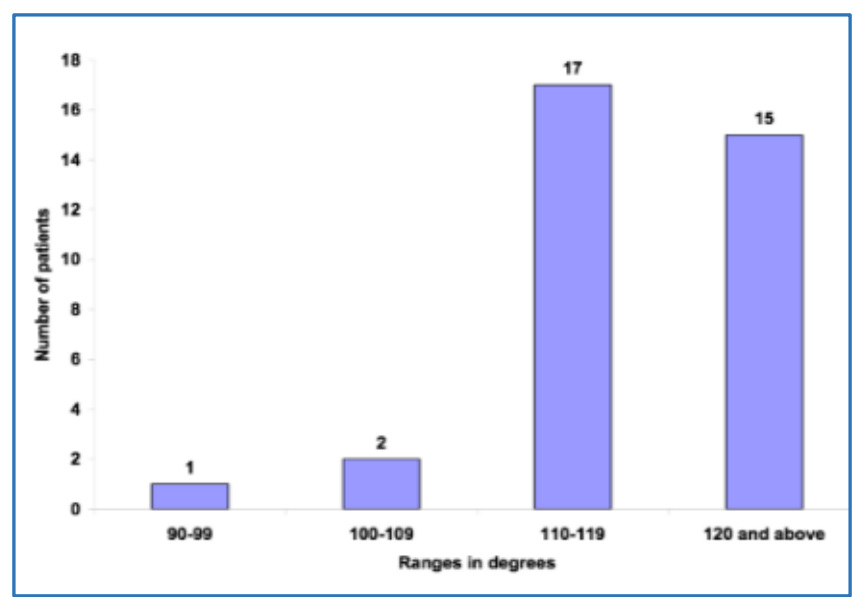

Fig. 2: Range of Motion Post-Operative

\section{Walking Distance}

Almost all patients had improved walking distance when compared to preoperative period. Walking distance improved significantly.

\section{Figure: Walking Distance}

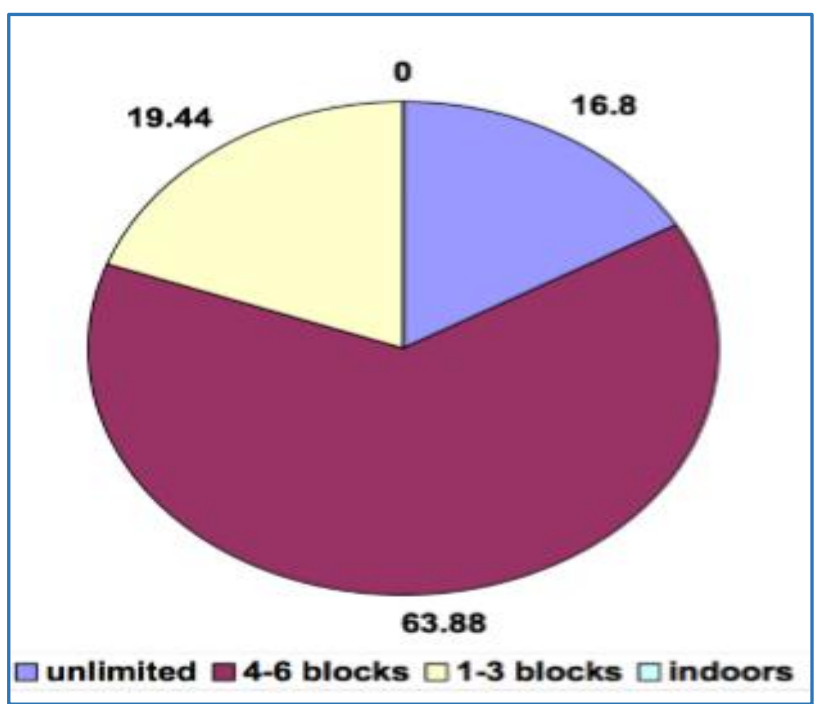

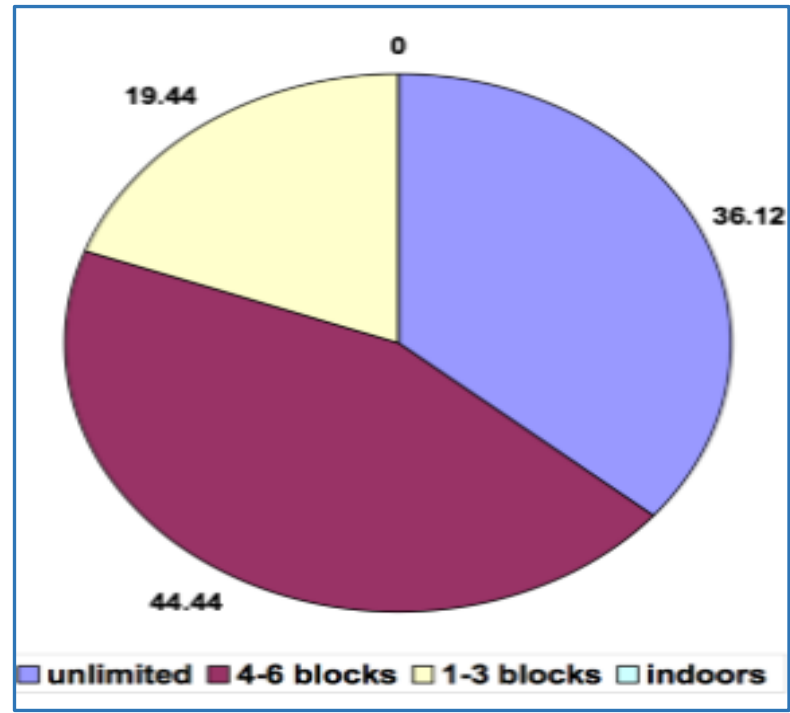

Tibiofemoral Angle

Postoperative tibio femoral angle which was aimed for over correction was assessed.

\begin{tabular}{|c|c|c|c|}
\hline $\begin{array}{c}\text { No. of } \\
\text { Patients }\end{array}$ & $\begin{array}{c}\text { Preop } \\
\text { tibiofemoral } \\
\text { angle in } \\
\text { Degrees }\end{array}$ & $\begin{array}{c}\text { No. of } \\
\text { Patients }\end{array}$ & $\begin{array}{c}\text { Postop } \\
\text { tibiofemoral } \\
\text { angle in } \\
\text { Degrees }\end{array}$ \\
\hline 20 & $0-4^{0}$ & 2 & $0-4^{0}$ \\
\hline 11 & $5-9^{0}$ & 24 & $5-9^{0}$ \\
\hline 5 & $10-15^{0}$ & 10 & $10-15^{0}$ \\
\hline \multicolumn{3}{|c|}{ Table 1: Post-operative tibiofemoral angle } \\
\hline
\end{tabular}

\section{Complications}

\begin{tabular}{|c|c|c|}
\hline Complications & No. of Patients & Percentage \\
\hline Infection & 1 & 2.78 \\
\hline Non-union & 1 & 2.78 \\
\hline Ext-lag & 1 & 2.78 \\
\hline None & 33 & 91.67 \\
\hline \multicolumn{3}{|c|}{ Table 2: Complications } \\
\hline \multicolumn{3}{|c|}{} \\
\hline
\end{tabular}

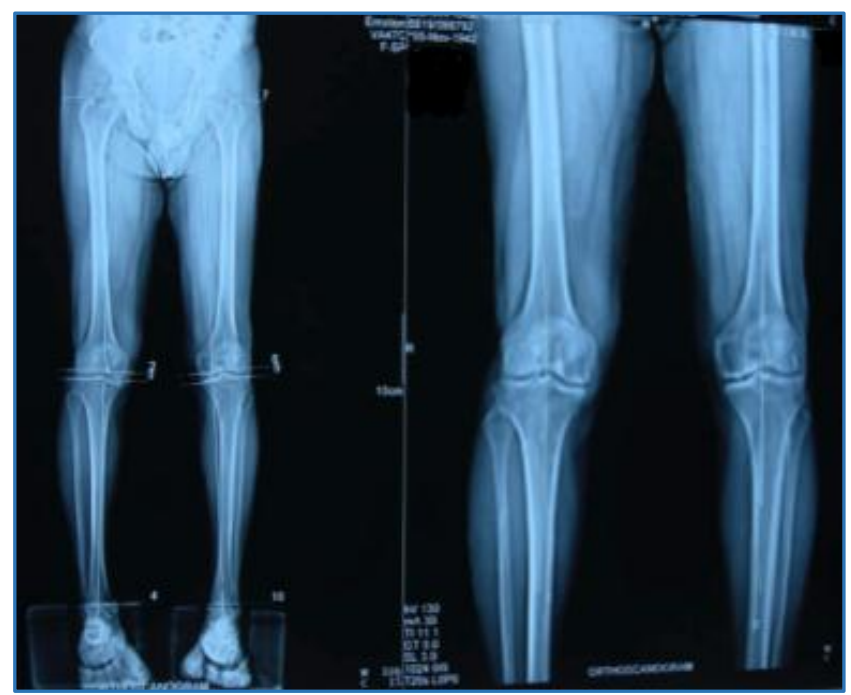

Preoperative Orthoscanogram 


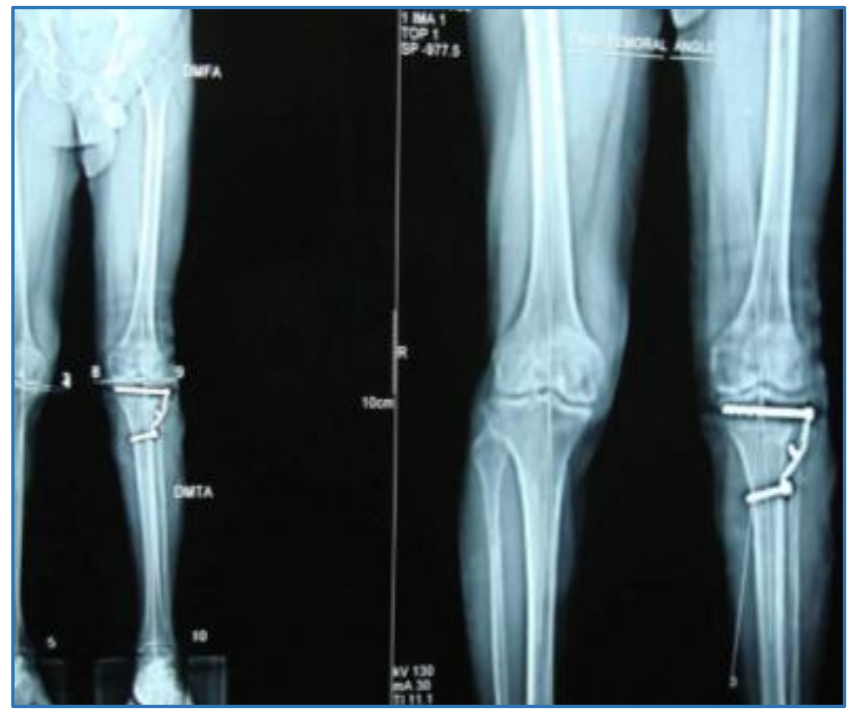

Postoperative Orthoscanogram

\section{DISCUSSION}

In the treatment of osteoarthrosis, the goals are to reduce the pain, shift the mechanical axis and improve the function. In manual laborers who are economically backward and cannot afford the latest implants for unicompartmental knee replacement, this is a valuable tool to treat medial compartmental arthritis

High tibial osteotomy is an excellent modality in treating unicompartmental osteoarthrosis.

Our study includes thirty six patients treated at our hospital.

\section{Sex Distribution}

In our study twenty four patients were female and twelve patients were male, where as in Coventry ${ }^{3}$ study forty eight patients were male and twenty five were female, in Insall's ${ }^{4}$ study twenty four patients were male and fifty nine patients were female. More number of female patients was noted in our study and Insall ${ }^{4}$ study when compared to Coventry's. ${ }^{3}$ study. Insall. ${ }^{4}$ recommended that high tibial valgus osteotomy is reserved for the patients younger than sixty years of age.

Coventry. ${ }^{3}$ suggested that the age of the patient is irreverent for surgery and the average age of patients in the series is forty nine years. In our study we feel that osteotomy should not be denied to patients based on the age if the other criteria like unicompartmental involvement, stable joint with no fixed deformities are present. The mean age of patients' in our study is 54.44 years. The mean ages of Insall's. ${ }^{4}$ and Coventry. $^{3}$ studies were sixty years and sixty three years respectively.

\section{Osteotomy and Instrumentation}

All thirty six patients had undergone lateral closed wedge osteotomy with tibio fibular syndesmotic release.

Opening wedge osteotomy is indicated in early, medium and advanced arthritis, in the older group, up to 70 years, and also if a large correction is needed. This opening technique allows easier placement of an eventual secondary TKA. It also has less neurovascular complication risks.

Closing wedge osteotomy in medium varus angles, in younger patients who generally have good bone stock whereas Insall. ${ }^{4}$ and Coventry. ${ }^{3}$ has done lateral closed wedge osteotomy with fibular head excision.
In our study we used cancellous screw with washer and a cortical screw, SS wire in the form of figure of eight. Coventry. ${ }^{3}$ used staples for closure of osteotomy. Insall. ${ }^{4}$ haven't used any instrumentation. Kazunori Yasuda etal. ${ }^{5}$ used closed wedge osteotomy and fixation with charnley external fixation device. Aaron. A Hofmann et al. ${ }^{6}$ used transverse tibial jig for osteotomy and one buttress plate used for fixation. Krackow. ${ }^{7}$ used using paediatric blade staple for internal fixation. Lemaire. ${ }^{8}$ reported rigid fixation with angled blade plate. Billings et al. ${ }^{9}$ had done calibrated osteotomy transverse osteotomy jig and used one buttress plate. When compared to the above studies we have used minimal instrumentation.

\section{Pain}

The mechanical pain was assessed as none, mild, moderate and severe. In our study the number of patients without pain postoperatively is thirty two (94.44\%), and patients with mild pain were three $(5.56 \%)$ and severe pain was one $(2.78 \%)$ patient respectively. Insall. ${ }^{4}$ reported $97 \%$ with no pain and $3 \%$ had mild pain. In Coventry. ${ }^{3}$ study $67 \%$ patients had mild pain, $24 \%$ had moderate pain and $7 \%$ had severe pain during followup.

Pain either disappeared or was relieved to a large extent after surgery. Arnoldi. ${ }^{10}$ felt that the relief of pain was due to lowering of the intraosseous venous pressure. Coventry agreed with this hypothesis that osteotomy may lower the intraosseous hypertension in the short term. The mechanical realignment of the joint with the transfer of load from the involved compartment to the opposite normal compartment is explained as the reason for relief of pain.

\section{Range of Motion}

In our study there was 5 degrees improvement in mean range of motion on average. None of the patients lost any further flexion in final follow up where as in Insall. ${ }^{4}$ study the range of motion was decreased in six patients. In Coventry's. ${ }^{3}$ study the range of motion decreased by a mean of 5 degrees and there was 5 degrees of mean flexion and 5 degrees of mean extension lost in the final follow up. From the above data there was improvement in range of motion in our study when compared to the above studies may be due to early mobilization when compared to the above studies which were immobilized in plaster cast.

\section{Walking Distance}

In our study almost all patients had improved walking distance when compared to preoperative period. Preoperatively $19.44 \%$ patients were able to walk 1 to 3 blocks and patients walking 4 to 6 blocks were $63.88 \%$ and those walking unlimited were $16.88 \%$ whereas after surgery patients walking 4 to 6 blocks was increased to $44.4 \%$ and patients walking unlimited was $36.2 \%$. In Coventry. ${ }^{3}$ study patients walking from 1 to 3 blocks were $44 \%$, 4 to 6 blocks were $34 \%$ unlimited were $16 \%$ post operatively those walking 1 to 3 blocks reduced to $19.4 \%$ and those walking 4 to 6 blocks rose to $44.4 \%$, those walking unlimited rose to $36.12 \% .80 .6 \%$ of the patients had improved walking distance and $16.62 \%$ patients had same walking distance as earlier and $2.78 \%$ patients had reduced walking distance whereas in Coventry. ${ }^{3}$ study, $54 \%$ had better walking distance post operatively and $37 \%$ patients had same walking distance as before the surgery and $9 \%$ patients had reduced walking distance after the surgery. 
The improvement of walking distance in our patients is due to stable fixation and early mobilization.

\section{Tibiofemoral Angle}

Postoperatively tibiofemoral angle was measured with radiographs and orthoscanograms. In our study the postoperative tibiofemoral angles were assessed in percentage. $66.6 \%$ patients had tibiofemoral valgus angles of 5 to 9 degrees. $27.78 \%$ patients had between 10 to 15 degrees of valgus. In Insall study $43.96 \%$ patients were in the ranges of 5 to 9 degrees and $45.06 \%$ patients were in the range of 10 to 15 degrees. In Coventry. ${ }^{3}$ study $98 \%$ of knees were in valgus angulation at final follow up. From the above data, in our study we have achieved $94.43 \%$ of desired or over correction of tibiofemoral angle whereas in Insall's. ${ }^{4}$ study it was $98.46 \%$.

The rationale of proximal tibial osteotomy is to change the varus deformity to valgus angulation at the knee and thereby decreasing the load on the medial plateau. The question is how much valgus angulation is correct amount. Coventry. ${ }^{3}$ recommended 5 degrees over correction beyond the normal angle of 5 to 8 degrees of genu valgum or a final tibiofemoral valgus angle of 10 to 13 degrees.

Paley. ${ }^{11}$ et al. eventually concluded that 2 to 4 degrees of mechanical valgus over correction achieved the best results whereas neutral alignment, under correction or significant over correction led to inferior results.

Bauer recommended correction up to a tibiofemoral valgus angle of 3 to 16 degrees. We found that the over correction should be at least 2-4 degrees of valgus, but cannot specify an upper limit with certainty. This finding suggests that there is individual variation. We had, at final follow-up, an average tibiofemoral valgus angulation of 6.6 degrees. Keeping the results in view, we noted good results if an average tibiofemoral valgus angulation measured 8.5 degrees, two knees rated poor in our series had valgus angulation less than 2 degrees. Insall. ${ }^{4}$ reported that the best results were obtained in knees which had tibiofemoral angle between 10 to 14 degrees post operatively. They felt that post-operative valgus angle between 5 degrees and 14 degrees to be acceptable. Paley. ${ }^{11}$ feels 2 to 4 degrees over correction in other words $\left(5^{\circ}+2^{\circ}=7^{\circ}\right)$ or $\left(5^{\circ}+4^{\circ}=9^{\circ}\right) 7$ to 9 degrees of valgus angulation is ideal.

\section{CONCLUSION}

High tibial osteotomy remains a useful and efficient procedure. The improvement in the post op scoring encourages us to continue recommending HTO in the most painful early and medium stages of medial compartment osteoarthritis.

HTO is thus very beneficial in cases of a good patient selection and good operative technique has been achieved. In most cases, this osteotomy is good enough to avoid or delay knee arthroplasty or at least diminish their number.

\section{REFERENCES}

1. Sherman C, Cabanela M (2009). Closing wedge osteotomy of the tibia and the femur in the treatment of gonarthrosis. Int Orthop. doi:10.1007/s00264-009-0883-1.

2. Amendola A, Panarella L (2005). High tibial osteotomy for the treatment of unicompartmental arthritis of the knee. Orthop Clin North Am 36(4):497-504.

3. Coventry m.b.: upper tibial osteotomy for gonarthrosis. The evolution of operation in the last 18 years and long term results. Orthop clin North America, 10.1: 191-210, 1979.

4. Insall JN, Joseph DM, Msika C: high tibial osteotomy for varus gonarthrosis. J Bone and Joint Surg, 66-a: 1040-1047, Sept. 1984.

5. Kazunori Yasuda.: A 10 to 15 year follow-up of high tibial osteotomy in medial compartment arthritis. Clinical orthopedics and related research 186-95, 10/1992.

6. Hofmann, Aaron A, MD; Wyatt, Ronald WB, MD; Beck Scott W, MD. Clinical orthopaedics and related research. 271:212-217, October 1991.

7. Krackow, Kenneth A, MD; Holtgrewe, Jeffrey L, MD. Clinical orthopaedics and related research. 258:213-224, September 1990.

8. Lemaire R. Comparative study of 2 series of tibial osteotomies with blade plate fixation or compression frame. Acta Orthop Belg, 48, 157-171, 1982.

9. Billings A, David F Scott, Marcelo P, et al. High tibial osteotomy with a calibrated guide, rigid internal fixation and early mobilization. J Bone Joint Surgery 2000 Jan; 82(1):70- 79 .

10. Arnoldi CC, Lemperg RK and Lindeholm. Intraosseous hypertension and pain in the knee. J Bone and Joint Surg, 57-b (3):360-363, 1975.

11. Paley D, Maar DC, Herzenberg JE. New concepts in high tibial osteotomy for medial compartment osteoarthritis. Orthop Clin North Am, 1994 Jul; 25(3):483-98. 